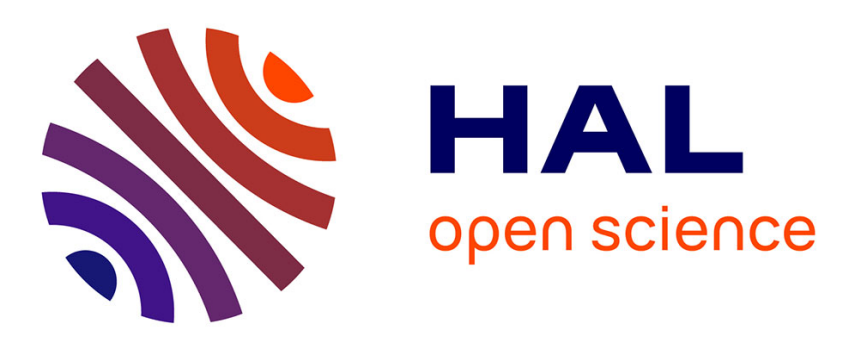

\title{
Conical intersections induced by light: Berry phase and wavepacket dynamics
}

Gábor J Halász, Ágnes Vibók, Milan Šindelka, Nimrod Moiseyev, Lorenz S Cederbaum

\section{- To cite this version:}

Gábor J Halász, Ágnes Vibók, Milan Šindelka, Nimrod Moiseyev, Lorenz S Cederbaum. Conical intersections induced by light: Berry phase and wavepacket dynamics. Journal of Physics B: Atomic, Molecular and Optical Physics, 2011, 44 (17), pp.175102. 10.1088/0953-4075/44/17/175102 . hal00651651

\section{HAL Id: hal-00651651 https://hal.science/hal-00651651}

Submitted on 14 Dec 2011

HAL is a multi-disciplinary open access archive for the deposit and dissemination of scientific research documents, whether they are published or not. The documents may come from teaching and research institutions in France or abroad, or from public or private research centers.
L'archive ouverte pluridisciplinaire HAL, est destinée au dépôt et à la diffusion de documents scientifiques de niveau recherche, publiés ou non, émanant des établissements d'enseignement et de recherche français ou étrangers, des laboratoires publics ou privés. 


\title{
Conical intersections induced by light: Berry phase and wave packet dynamics
}

\author{
Gábor J. Halász ${ }^{1,2,5}$, and Ágnes Vibók ${ }^{2,3,5}$ \\ ${ }^{1}$ Department of Information Technology, University of Debrecen, \\ H-4010 Debrecen, PO Box 12, Hungary \\ ${ }^{2}$ Kavli Institute for Theoretical Physics, \\ University of California, \\ Santa Barbara, CA 93106-4030, USA \\ ${ }^{3}$ Department of Theoretical Physics, \\ University of Debrecen, \\ H-40410 Debrecen, PO Box 5, Hungary \\ Milan Šindelka ${ }^{4}$ and Nimrod Moiseyev ${ }^{4}$ \\ ${ }^{4}$ Schulich Faculty of Chemistry, Department of Physics, \\ and Minerva Center of Nonlinear Physics in Complex Systems, \\ Technion - Israel Institute of Technology, \\ Haifa 32000, Israel \\ Lorenz S. Cederbaum ${ }^{2,5}$ \\ ${ }^{5}$ Theoretische Chemie, Physikalisch-Chemisches Institut, \\ Universität Heidelberg, H-69120, Germany

\begin{abstract}
Conical intersections (CIs) play an important role in the nonadiabatic molecular processes. Characterizing and localizing them is important for describing and controlling electronic energy flow in molecules. It is known that no conical intersection appears in free diatomic systems. In earlier works [J. Phys. B. 41, 221001 (2008), J. Phys. B. 44, 045603 (2011)] it was pointed out that CIs can be formed both by standing and running laser waves even in diatomics. The energetic and internuclear positions of these CIs depend on the laser frequencies while the strength of their nonadiabatic couplings can be modified by the field intensities. In this work we calculate the topological or Berry phase of the light-induced $\mathrm{CI}$ in the $\mathrm{Na}_{2}$ molecule. The presence of this phase is a clear fingerprint of the laser-induced CI. In addition, we perform a detailed study of the wave packet propagation and discuss effects which reflect the significant presence of the laser-induced CI.
\end{abstract}

\section{Introduction}

Conical intersections (CIs) between electronic potential energy surfaces play a key mechanistic role in the nonadiabatic molecular processes [1-3]. In this case the nuclear and electronic motion can couple and the energy exchange between the electrons and nuclei may become significant. In several important cases like dissociation, proton transfer, isomerization processes of polyatomic molecules or radiationless deactivation of the excited state systems [4-9] the CIs can provide very efficient channel for ultrafast interstate crossing on the femtosecond time scale. CIs can be evolved between different electronic states starting from triatomic systems to a truly large polyatomic molecules. Several important books, review articles and publications have demonstrated the existence and relevance of such intersections in recent decades [3, 10-12].

Generally CIs are not isolated points in the configuration space. Rather, they are an infinite number of connected points forming a seam. For a diatomic molecule that has only one degree of freedom, it is not possible for two electronic states of the same symmetry to become degenerate and as a consequence of the well-known noncrossing rule an avoided crossing results. It should be noted, however, that this statement is true only in free space. As it was shown in earlier papers $[13,14]$ CIs can be formed both by standing and running laser waves even in diatomic systems. In the former case the laser light-induces CIs which couple the center of mass motion with the internal rovibrational degrees of freedom. In the latter case, the rotational motion constitutes the missing degree of freedom to allow for the formation of a CI. It was found that in a diatomic system under the influence of a standing laser field (optical lattice) a periodic array of CIs is formed by the laser light [13]. In addition, it was shown that the appearance of these light-induced CIs can significantly reduce the magnitude of the trapping effect of cold diatomic molecules in the lowest electronic state. For the case of running laser waves, it was demonstrated that there is a very strong impact of the light-induced CIs on the molecular spectrum and spectral properties [14].

We would like to mention that often the rotational degree of freedom is neglected and only the vibrational one is taken into account in computing diatomic molecules in 
laser fields, and thus the CI is neglected (see e.g. [1517] and citations therein). Previous works demonstrated, however, the importance of the rotations in the presence of an intense laser field [18, 19].

The light-induced CIs constitute a novel and physically interesting new laser-matter phenomenon. The presence of these light-induced CIs in diatomic systems may completely change their original, i.e., field free, physical properties. In other words, using either standing or running laser waves it is possible to build in significant nonadiabatic effects into molecular systems using manipulable external forces allowing one to open up a new direction in the field of molecular quantum control processes.

The light-induced strong nonadiabaticity couples in a controllable way different electronic states of the molecule and depending on the field intensity can be extremely large in the vicinity of the CIs. The nonadiabatic couplings become singular at the CIs [10]. The presence of a CI provides the source for numerous statical and dynamical nonadiabatic phenomena. One of these phenomena is the appearance of a topological effect often referred to as Longuet-Higgins or Berry phase [21-23]. In this work as a continuation of the former ones [13, 14] we choose again the $\mathrm{Na}_{2}$ molecule as a sample system. One of our aims is to calculate the topological or Berry phase in $\mathrm{Na}_{2}$. The presence of this phase is a clear fingerprint of the laser-induced CI.

As well studied in free polyatomic molecules, the dynamics of the system is highly affected by CIs and becomes strongly nonadiabatic owing to the strong coupling between the nuclei and electrons. The importance of this feature cannot be overemphasized. Therefore, investigating the dynamics in the region of laser induced CI is another goal of this paper. The presented wave packet propagation study is expected to be of particular interest and to reflect the effect of the light-induced CI.

The paper is organized as follows. Section II gives a brief description of our working Hamiltonian. In Section III a short characterization of the CIs and of the resulting nonadiabatic couplings is presented. We present the Berry phase and the results of the wave-packet calculations related to the two lowest states of sodium dimer in Section IV and V, respectively. These sections are also devoted to the discussion. Conclusions are presented in Section VI.

\section{The Hamiltonian}

The results reported here are obtained by solving the time-dependent Schrödinger equation for the nuclear motion of $\mathrm{Na}_{2}$ proceeding on two coupled potential energy surfaces. The respective electronic states are coupled by a running laser wave of wavelength $\lambda$, and labeled $\mathrm{X}$ (ground, $X^{1} \Sigma_{g}^{+}$) and A (excited, $A^{1} \Sigma_{u}^{+}$):

$$
i \hbar \frac{d}{d t}\left(\begin{array}{c}
\psi_{X} \\
\psi_{A}
\end{array}\right)=\hat{\mathbf{H}}\left(\begin{array}{c}
\psi_{X} \\
\psi_{A}
\end{array}\right)
$$

where the total molecule and field Hamiltonian is

$$
\hat{\mathbf{H}}=\left(\hat{\mathbf{H}}_{v i b}+\hat{\mathbf{H}}_{r o t}\right)+\hat{\mathbf{W}} .
$$

Here, $\hat{\mathbf{H}}_{v i b}$ and $\hat{\mathbf{H}}_{r o t}$ are the molecular vibrational and rotational kinetic energy Hamiltonian, respectively, and $\hat{\mathbf{W}}$ is the potential energy matrix in the above two electronic states. These terms are given by

$$
\begin{gathered}
\hat{\mathbf{H}}_{v i b}=-\hat{\mathbf{1}} \frac{\hbar^{2}}{2 \mu} \frac{\partial^{2}}{\partial R^{2}}, \\
\hat{\mathbf{H}}_{r o t}=\hat{\mathbf{1}} \frac{\mathbf{L}_{\theta \varphi}^{2}}{2 \mu R^{2}},
\end{gathered}
$$

$\mu$ being the reduced mass of the diatomic molecule, $\mathrm{R}$ and $(\theta, \varphi)$ are its vibrational and rotational coordinates, respectively, and $\mathbf{L}_{\theta \varphi}^{2}$ is the angular momentum operator of the nuclei. The molecule-light term coupling the two electronic states can be written as the scalar product of the transition dipole moment $\vec{d}$ and the electric-field vector $\vec{\varepsilon}$ :

$$
\vec{d} \cdot \vec{\varepsilon}=\epsilon_{0} d(R) \cos \theta \cos \omega_{L} t
$$

Here $\epsilon_{0}$ is the maximum laser field amplitude, $\omega_{L}$ is the laser frequency which couples the two electronic states, $d(R)$ is the transition dipole matrix element. For symmetry reason only the $z$ component of the transition dipole moment $\left(d(R)=<\psi_{1}^{e}\left|\sum_{j} z_{j}\right| \psi_{2}^{e}>\right)$ is non-vanishing.

The potential part $\hat{\mathbf{W}}$ contains the field-free potential energy curves $V_{X}$ and $V_{A}$ of the two electronic states as diagonal elements and the radiative coupling as the nondiagonal element. In the static dressed state representation this potential energy matrix has the following form:

$$
\hat{\mathbf{W}}=\left(\begin{array}{cc}
V_{X} & \left(\epsilon_{0} / 2\right) d(R) \cos \theta \\
\left(\epsilon_{0} / 2\right) d(R) \cos \theta & V_{A}-\hbar \omega_{L}
\end{array}\right) .
$$

The potential energies $V_{X}$ and $V_{A}$ of the two electronic states and the transition dipole moment were taken from [24].

\section{Conical Intersection and Nonadiabatic Coupling Matrix}

In eq. (6) of Sec. II. we gave the expression for the potential energy matrix $\hat{W}$. To continue, we define as usual the unitary matrix $\hat{U}$ which diagonalizes the potential energy matrix and thus transforms the Hamiltonian (2) to its adiabatic representation: 


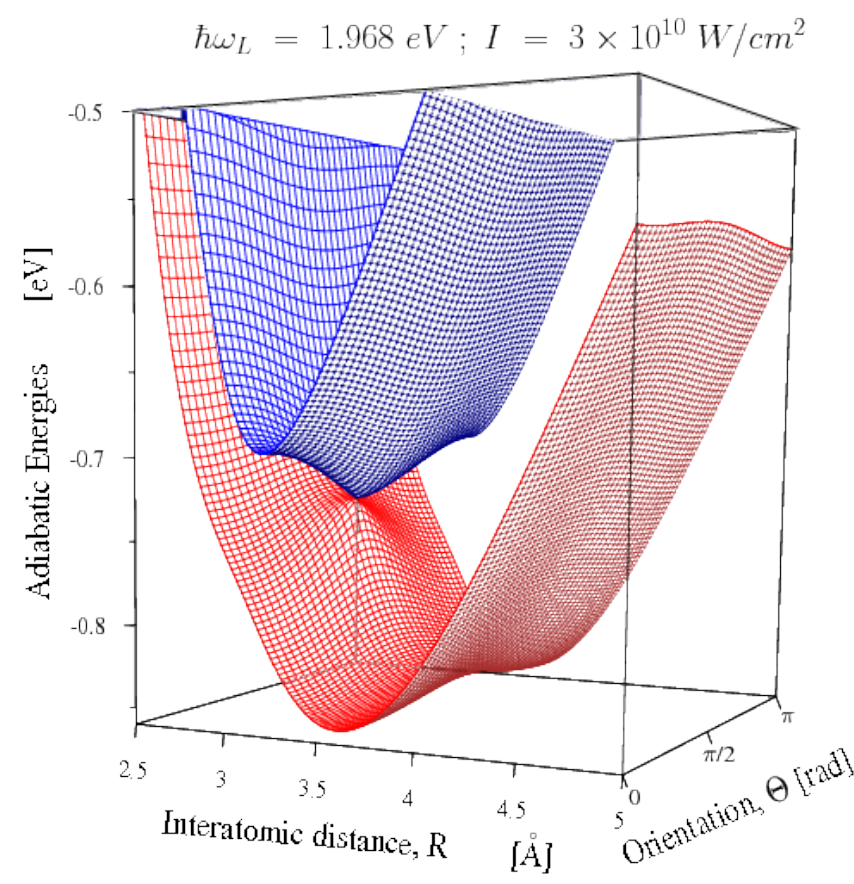

Figure 1: Light-induced conical intersection between the $V_{a d}^{\text {lower }}(R, \theta)$ and $V_{a d}^{u p p e r}(R, \theta)$ adiabatic potential energy surfaces in the $\mathrm{Na}_{2}$ molecule. The applied field intensity and photon energy are: $I=3.0 \times 10^{10} \frac{\mathrm{W}}{\mathrm{cm}^{2}}$ and $\hbar \omega_{L}=1.968 \mathrm{eV}$.

$$
U(R, \theta)=\left(\begin{array}{cc}
\cos \Phi(R, \theta) & \sin \Phi(R, \theta) \\
-\sin \Phi(R, \theta) & \cos \Phi(R, \theta)
\end{array}\right)
$$

with

$$
\Phi(R, \theta)=\frac{1}{2} \arctan \left(\frac{\varepsilon_{0} d(R) \cos \theta}{V_{A}(R)-\hbar \omega_{L}-V_{X}(R)}\right) .
$$

In this picture the $\hat{U} \hat{W} \hat{U}^{\dagger}$ transformation produces the adiabatic potential energy surfaces, $V_{a d}^{\text {lower }}(R, \theta)$ and $V_{a d}^{u p p e r}(R, \theta)$. These two potential energy surfaces can cross each other, forming a real conical intersection (see Figure 1), only if the two conditions $(\cos \theta=0,(\theta=\pi / 2)$ and $\left.V_{X}(R)=V_{A}(R)-\hbar \omega_{L}\right)$ are simultaneously fulfilled. The characteristic features of this CI can be changed by varying the frequency and intensity of the light field. Increasing the frequency, for example, moves the CI to a smaller internuclear distance and to a smaller energetic position. While the steepness of the CI cone formed by the adiabatic surfaces, which is related to the strength of the nonadiabatic coupling, can be controlled by the laser intensity.

The molecule-light couplings are eliminated from the potential energy matrix by applying the diagonalizing transformation specified above. On the other hand, nonadiabatic couplings now appear in the transformed kinetic energy operator. As is known from the literature
$[2,10]$, these nonadiabatic couplings can be calculated as the derivatives of the transformation angle $\Phi$ with respect to the corresponding nuclear coordinate

$$
\tau_{12 R}=\partial \Phi / \partial R \text { and } \tau_{12 \theta}=\partial \Phi / \partial \theta .
$$

In Figure 2 we display the nonadiabatic couplings in the close vicinity of the light-induced CI. As expected their values can be very large and diverge at the $\mathrm{CI}$ as is the case for natural CIs of polyatomics (compare with [10]). This leads us to conclude that the light-induced CIs introduce similar nonadiabatic effects as in polyatomics such that the usual Born-Oppenheimer approximation will not be valid any more.

\section{Topological or Berry Phase}

Having discussed some important features of the lightinduced CIs, let us study now the impact of this intersection on physical properties of the $\mathrm{Na}_{2}$. To continue we turn to the topological or Berry phase.

It was first pointed out by Longuet-Higgins and Herzberg [21, 22] that each real adiabatic electronic state changes sign when transported continuously along a closed loop enclosing the point of conical intersection. Mead and Truhlar connected this geometric phase effect with the single electronic state problem [25] and Berry generalized the theory [23]. Hence the name Berry phase. As the total wave function must be single valued, it is favorable to make the electronic wave function in the adiabatic representation complex by multiplying it by a phase factor ensuring that the total wave function remains single valued. The fact that the electronic eigenfunctions are modified has a direct effect on the nuclear dynamics even when a single potential energy surface is considered. Consequently, the appearance of the Berry phase in a molecular system can be considered as a clear signature of the conical intersection independently of whether it is a natural or a laser-induced one.

To continue, we refer to the literature [2] that the topological or Berry phase $\alpha_{12}$ can be calculated for a closed contour $\Gamma$ as:

$$
\alpha_{12}=\oint_{\Gamma} \tau_{12}\left(s^{\prime}\right) \cdot d s^{\prime}
$$

where $\tau_{12}$ is the non-adiabatic coupling term between the two electronic states, the components of which are introduced in the previous section. As a consequence of sign changes of the adiabatic states, it can be proven that the value of this phase is equal to:

$$
\begin{gathered}
\alpha_{12}=\pi \times\left\{\begin{array}{cc}
2 n+1 & \text { if } \Gamma \text { encircles odd number of CIs } \\
2 n & \text { if } \Gamma \text { encircles even number of CIs }
\end{array}\right. \\
n=0, \pm 1, \pm 2, \ldots
\end{gathered}
$$



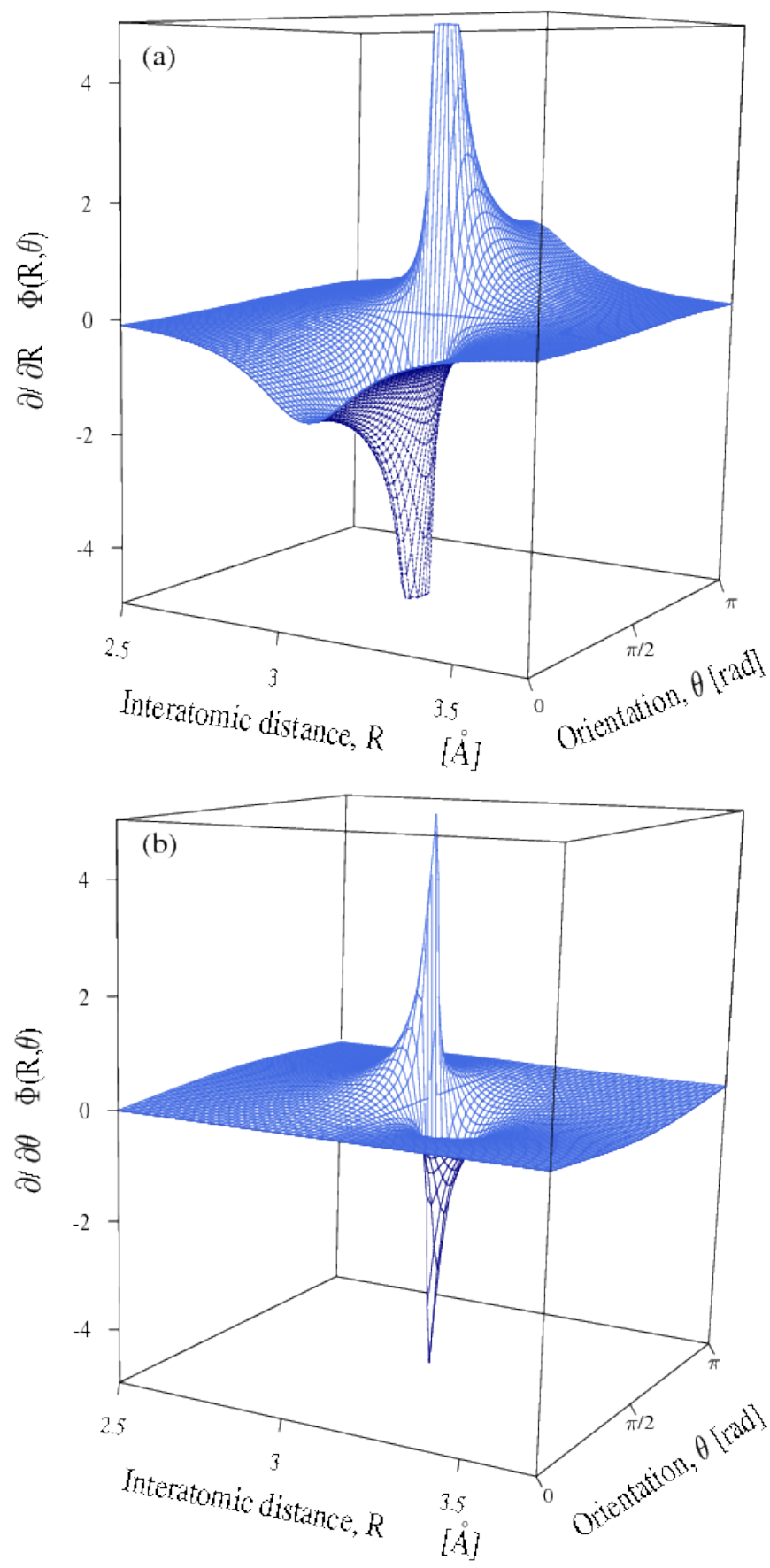

Figure 2: The nonadiabatic coupling terms $\left(\tau_{12 R}=\right.$ $\partial \Phi / \partial R$ and $\left.\tau_{12 \theta}=\partial \Phi / \partial \theta\right)$ for $\mathrm{Na}_{2}$. The applied field intensity and photon energy are: $I=3.0 \times 10^{10} \frac{\mathrm{W}}{\mathrm{cm}^{2}}$ and $\hbar \omega_{L}=$ $1.968 \mathrm{eV}$.

Now, using eq. (9), it is possible to calculate the Berry phase $\alpha_{12}$ for a closed path $\Gamma$ as follows:

$\alpha_{12}=\Phi(s 0)_{\text {end of the path }}-\Phi(s 0)_{\text {begining of the path }}$

In Fig. 3a we display the energy curve $V_{X}$ of the elec-
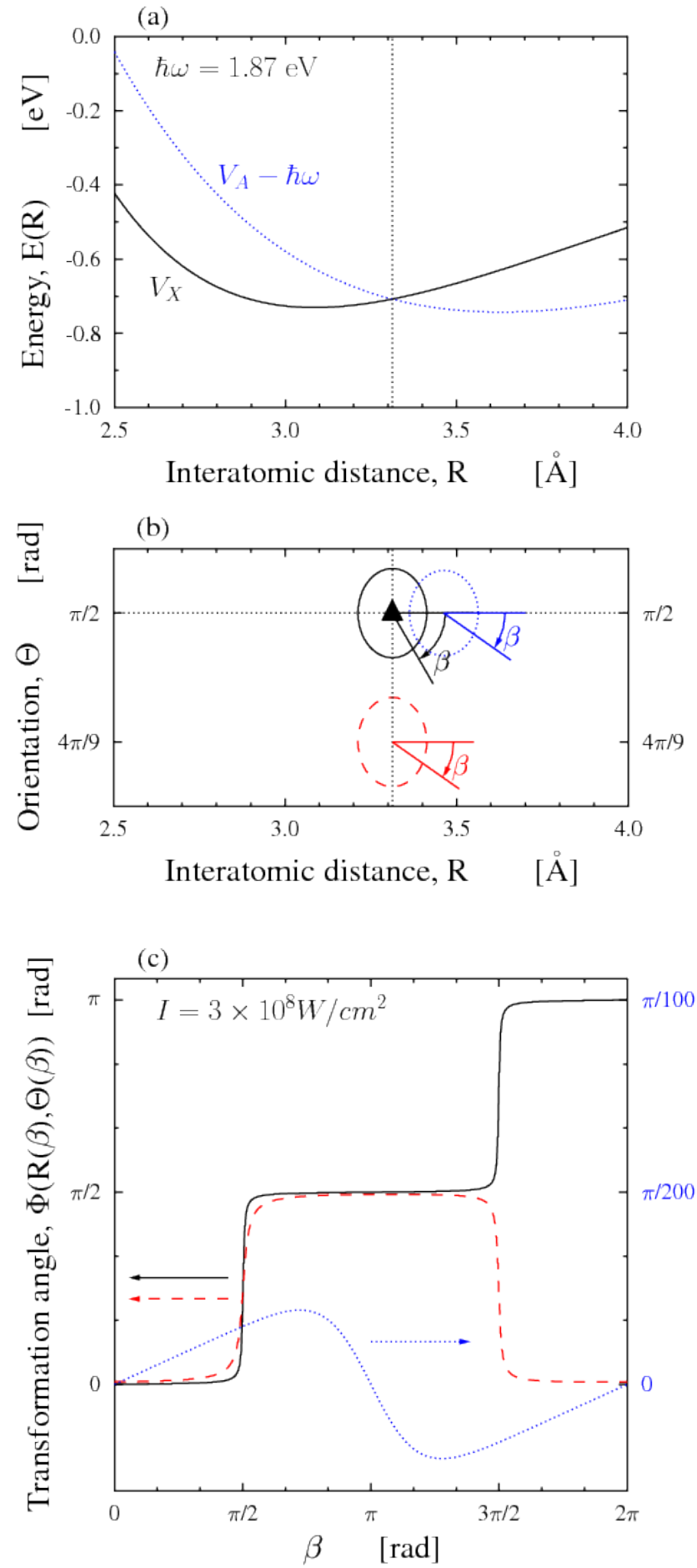

Figure 3: (a) Potential energy $V_{X}$ and field-dressed potential energy $V_{A}-\hbar \omega_{L}$ curves of $\mathrm{Na}_{2}$. The $\mathrm{X}$ and A notations correspond to the $X^{1} \Sigma_{g}^{+}$and $A^{1} \Sigma_{u}^{+}$electronic states. (b) Geometrical arrangement of the contours used in the topological phase calculations. The angle $\beta\{0 \ldots 2 \pi\}$ parametrizes the actual position in the configuration space where the transformation angle is calculated. The black triangle shows the position of the laser-induced conical intersection. (c) Transformation angles as a function of $\beta$ for three different geometrical arrangements. Only the solid curve is calculated along a path surrounding the CI. The arrows denote that the solid and dashed functions are given by the scale on the left side while the value of the dotted function is given by the scale on the right side. 
tronic ground state $X^{1} \Sigma_{g}^{+}$and the shifted energy curve $\left(V_{A}-\hbar \omega_{L}\right)$ of the first excited electronic state $A^{1} \Sigma_{u}^{+}$. The position of the curve crossing is indeed the position of the CI where the two conditions $(\cos \theta=0,(\theta=\pi / 2)$ and $\left.V_{X}(R)=V_{A}(R)-\hbar \omega_{L}\right)$ are satisfied. The geometrical arrangement used as contours in the topological phase calculations are shown in Fig. 3b. Here, three different circles are presented but only one of them surrounds the CI. The numerical calculations take place along these closed paths characterized by their centers $\left(R_{0}, \theta_{0}\right)$ and "radii" $\rho$. The actual position is given by an angle $\beta$ $\{0 \ldots 2 \pi\}:$

$$
\begin{aligned}
R & =R_{0}+\rho \cos \beta \\
\theta & =\theta_{0}-\frac{2 \rho}{R_{0}} \sin \beta .
\end{aligned}
$$

The field intensity is chosen as $I=3.0 \times 10^{8} \frac{\mathrm{W}}{\mathrm{cm}^{2}}$ at a photon energy of $\hbar \omega_{L}=1.870 \mathrm{eV}$. In Fig. 3c the obtained values of the $\Phi$ transformation angles are presented for the three arrangements shown. The Berry phase $\alpha_{12}$ can be calculated from the difference of $\Phi$ at the beginning and at the end of the path. It can be clearly seen that $\alpha_{12}$ differs from zero only in that situation, when the circle surrounds the CI. In this case the phase takes on the value $\alpha_{12}=\pi$. If a contour in a given plane does not surround the CI, the value $\alpha_{12}=0$ is found. These numerical results are in full agreement with eq. (11). We note, however, that the different curves display different shapes in Fig. $3 \mathrm{c}$ and all of them possess relatively sudden jumps along the path. This finding, although not affecting our conclusion concerning the encircling of a CI, is of interest too and may affect the dynamics of the single valued state mentioned above.

The results obtained for the transformation angle $\Phi(R, \theta)$ as a function of both coordinates $R$ and $\theta$ are displayed as color mapped pictures in Fig. 4 for two different intensities, $I=3.0 \times 10^{8} \frac{\mathrm{W}}{\mathrm{cm}^{2}}$ (Fig. 4a) and $I=3.0 \times 10^{10} \frac{\mathrm{W}}{\mathrm{cm}^{2}}$ (Fig. $4 \mathrm{~b}$ ). It is seen that the transformation angle changes when modifying the intensity of the laser field but, as expected, this does not cause any change in the value of the topological phases themselves for the closed contours. The sharpness of the transitions between different intervals is strongly affected by modifying the intensities but not the differences between the starting and the ending values of the transformation angle for closed contours.

\section{Wave Packet Dynamics}

In this section we discuss the impact of the lightinduced CI on the time-dependent wave packet propagation. We demonstrate that the appearance of lightinduced conical intersections leads to substantial and non-trivial effects in the dynamics. As is well known from the studies of conical intersections in polyatomics, the details of the dynamics are highly intricate and cannot be understood without explicit computations. It is in

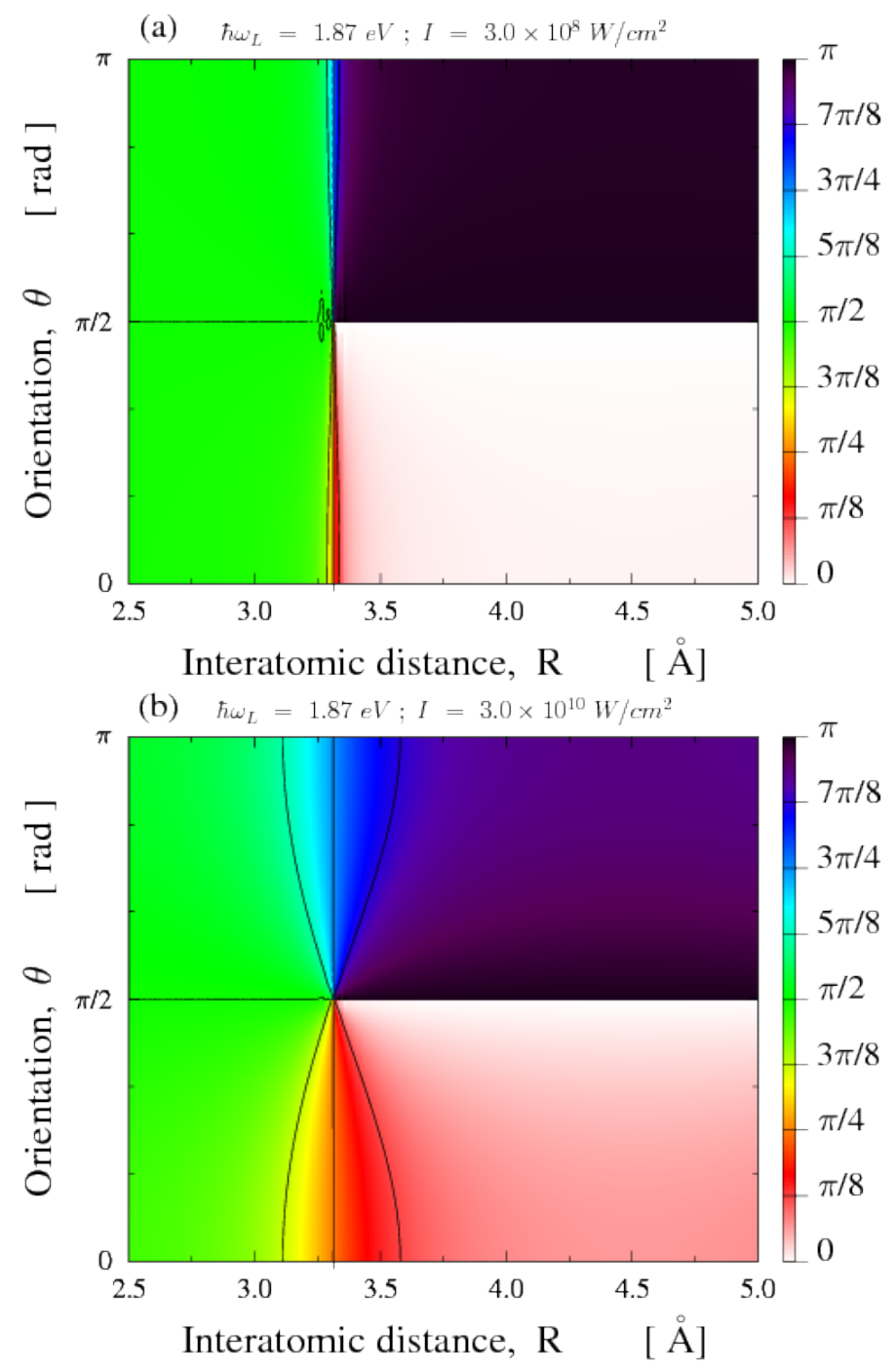

Figure 4: Color mapped plots of the transformation angle as a functions of $R$ and $\theta$ for two intensities of the laser field: (a) $I=3.0 \times 10^{8} \frac{\mathrm{W}}{\mathrm{cm}^{2}}$ and (b) $I=3.0 \times 10^{10} \frac{\mathrm{W}}{\mathrm{cm}^{2}}$.

general useless to try to relate details of the complex multimode dynamics to details of the nonadiabatic couplings. The situation is similar in the present case. The lightinduced CIs studied here lead to strong nonadiabatic mixing of rotational and vibrational states on both electronic adiabatic (or diabatic) surfaces which cannot be predicted without explicit computations. Several trends, however, can be understood from the general concept of CIs.

The results presented here are obtained by solving numerically the time-dependent Schrödinger equation (1). The evolution of the wave function is calculated by using the usual time propagator

$$
\left|\psi(R, \theta, t)>=\exp \left(-\frac{i}{\hbar} \widehat{H} t\right)\right| \psi(R, \theta, 0)>,
$$

where the time-independent Hamiltonian $\hat{\mathbf{H}}$ is given by eqs.(2) and (6). We expressed this Hamilto- 
nian in matrix representation in the basis set of the free molecular electronic and rovibrational states $\left(\left(\mid \varphi_{\nu, J}^{X}(R, \theta)>; 0\right)^{T}\right.$ and $\left.\left(0 ; \mid \varphi_{\nu, J}^{A}(R, \theta)>\right)^{T}\right)$. The spatial components of these free molecular states were calculated using the particle in a box functions, $\{\sqrt{2 / L} \sin (k \pi R / L)\}_{k=1,2, \ldots, N_{b}}$ which are multiplied by Legendre polynomials $\left\{P_{j}(\cos \theta)\right\}_{j=0,1,2, \ldots, N_{J}}$. In our numerical calculations we have used $N_{b}=500$ and $N_{J}=95$ for the X surface whereas for the A surface we have considered correspondingly 96 Legendre polynomials. In this representation the eigenvalues $\left(E_{j}\right)$ and eigen functions $\left(\phi_{j}\right)$ of the Hamiltonian can be obtained numerically. Next, one can describe the initial wave function in terms of these eigen functions: $|\psi(R, \theta, t=0)\rangle=\sum_{j} c_{j} \phi_{j}$. Based on this partitioning of the initial wave function one can reformulate equation 14 :

$$
|\psi(R, \theta, t>0)\rangle=\sum_{j} \exp \left(-\frac{i}{\hbar} E_{j} t\right) c_{j} \phi_{j} .
$$

Throughout the paper the initial wave function is chosen as the electronic and rovibrational ground state solution of the field free Hamiltonian: $|\psi(R, \theta, t=0)\rangle=$ $\left(\left|\varphi_{\nu=0, J=0}^{X}(R, \theta)\right\rangle ; 0\right)^{T}$.

To shed more light on the meaning of the wave packet propagation in the present context we stress that we are calculating the dynamics of a system which is first in a field free space and then suddenly exposed to the running laser wave. It is shown in the Appendix that this physical situation amounts to propagating the initial field free molecular state with the Hamiltonian (2-6). In order to compare with experiments, it suffices that in the latter the time of switching on the wave at the molecular site must be much shorter than the period of the laser.

The solution of eq.(14) is used to calculate the autocorrelation function which is the overlap between the initial and the time evolved wave packets

$$
\begin{aligned}
C(t) & =|<\psi(R, \theta, 0)| \psi(R, \theta, t)>\mid \\
& =\left|\int_{0}^{\pi} d \theta \cdot \sin \theta \int_{0}^{\infty} d R \cdot \psi(R, \theta, 0)^{*} \cdot \psi(R, \theta, t)\right| .
\end{aligned}
$$

as well as the time-dependent population on different adiabatic or diabatic surfaces $(S F)$

$$
\begin{aligned}
P^{S F}(t) & =<\psi^{S F}(R, \theta, t) \mid \psi^{S F}(R, \theta, t)>= \\
& \int_{0}^{\pi} d \theta \cdot \sin \theta \int_{0}^{\infty} d R \cdot \psi^{S F}(R, \theta, t)^{*} \cdot \psi^{S F}(R, \theta, t) .
\end{aligned}
$$

More specifically, we study the following quantities $P_{d i a}^{X}(t), P_{d i a}^{A}(t)$ and $P_{a d}^{u p p e r}(t)$, which correspond to the population of (or probability of being on) the ground state diabatic surface $(S F=X)$, on the excited state diabatic surface $(S F=A)$ and on the adiabatic upper state surface $(S F=$ upper $)[30]$, respectively. In the numerical calculations the two field intensities $I=3.0 \times 10^{8} \frac{\mathrm{W}}{\mathrm{cm}^{2}}$ and $I=3.0 \times 10^{10} \frac{\mathrm{W}}{\mathrm{cm}^{2}}$, and the two different values of the photon energy $1.851 \mathrm{eV}$ and $1.968 \mathrm{eV}$ were used. The internuclear $(R)$ and the energetic $(E)$ positions of the light-induced conical intersections are easily determined (see below) and, of course, do not depend on the used laser intensity. The dynamical investigations performed can be classified according to the actual position of the conical intersections and intensity. To avoid numerous figures showing the many results obtained, we collect the numerical results for the autocorrelation and various population functions compactly in a single picture for each set of parameters (photon energy and field intensity).

We begin with the weak field situation $(I=3.0 \times$ $\left.10^{8} \frac{\mathrm{W}}{\mathrm{cm}^{2}}\right)$. In Fig. 5 we first display the results for $\hbar \omega_{L}=1.851 \mathrm{eV}$. Here, the energy of the initial wave packet is slightly above the bottom of the $V_{A}-\hbar \omega_{L}$ curve, but still below the lowest vibrational level of angular momentum $J=1$ on the A surface to which it couples directly. Furthermore, the energy of initial wave packet is also below that the energy of the CI. It is known that in such cases the nonadiabatic effects on the initial wave packet are weak [10]. Consequently, the whole wave packet stays localized on the $\mathrm{X}$ surface up to $50 \mathrm{ps}$ and the populations on the two diabatic surfaces remain practically unchanged. Turning to the adiabatic picture, the situation is similar. The population on the upper surface is more or less constant and very low (around $\sim 0.004$ ) apart from the first $1-2 \mathrm{ps}$. On the other hand, the autocorrelation function does show clear periodical changes which due to fact that the initial field free state is a linear combination of essentially two eigenfunctions of the full Hamiltonian $(2,6)$. Ordered by increasing energy, these are the first and third eigenstates $\phi_{1}$ and $\phi_{3}$, and are initially populated by $\sim 73.3 \%$ and $\sim 26.4 \%$, respectively.

We now increase the laser frequency to $\hbar \omega_{L}=$ $1.968 \mathrm{eV}$. The results of the computations are collected in Fig. 6 in analogy to Fig. 5. In this situation both the energetic and internuclear positions of the CI change to lower values. Importantly, the position of the initial wave packet is now higher by about $0.1 \mathrm{eV}$ than the bottom of the $V_{A}-\hbar \omega_{L}$ curve, and is also above the position of the CI. In contrast to the first example, the wave function can now spread out more efficiently through the CI. The decay of the autocorrelation function is rather fast: it arrives at its first zero after $1.34 \mathrm{ps}$. At later times it oscillates and exhibits a larger recurrence at around $9.5 \mathrm{ps}$ and thereafter continues to oscillate with a larger amplitude than before this recurrence. In this case the initial wave function is a linear combination of several eigenfunctions. Nine of them cover more than $98 \%$ of the initial wave function. The periods of times related to the energy differences between these eigenfunctions are in the range from $1.5 \mathrm{ps}$ to $20.1 \mathrm{ps}$ and five of them - between neighbouring states - are between $9.8 \mathrm{ps}$ and $10.9 \mathrm{ps}$. The interference of these states causes the "irregular" oscillations and the recurrences of the autocorrelation function. All populations show large variations with time. The population on the $\mathrm{X}$ surface is changing 

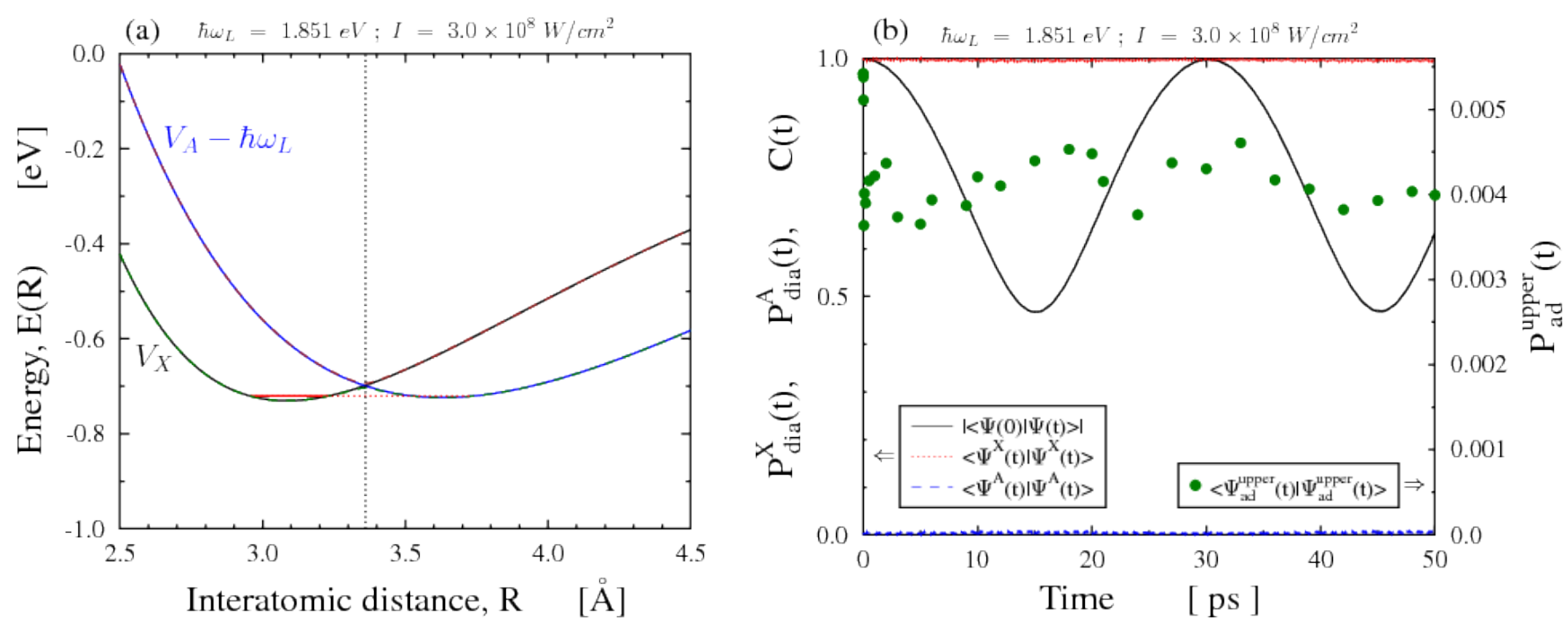

Figure 5: Potential curves and results for field intensity $I=3.0 \times 10^{8} \frac{\mathrm{W}}{\mathrm{cm}^{2}}$ and frequency $\hbar \omega_{L}=1.851 \mathrm{eV}$. (a) Potential energy $V_{X}$ and field-dressed potential energy $V_{A}-\hbar \omega_{L}$ curves of $\mathrm{Na}_{2}$. The dashed-dotted vertical line indicates the geometric position of the CI and the dashed-dotted horizontal line the energy position of the initial state of the propagation. (b) Autocorrelation function (solid line), population on the ground state diabatic surface (dotted line), on the excited state diabatic surface (dashed line) and on the adiabatic upper state surface $(\bullet \bullet)$ are shown as a function of time.
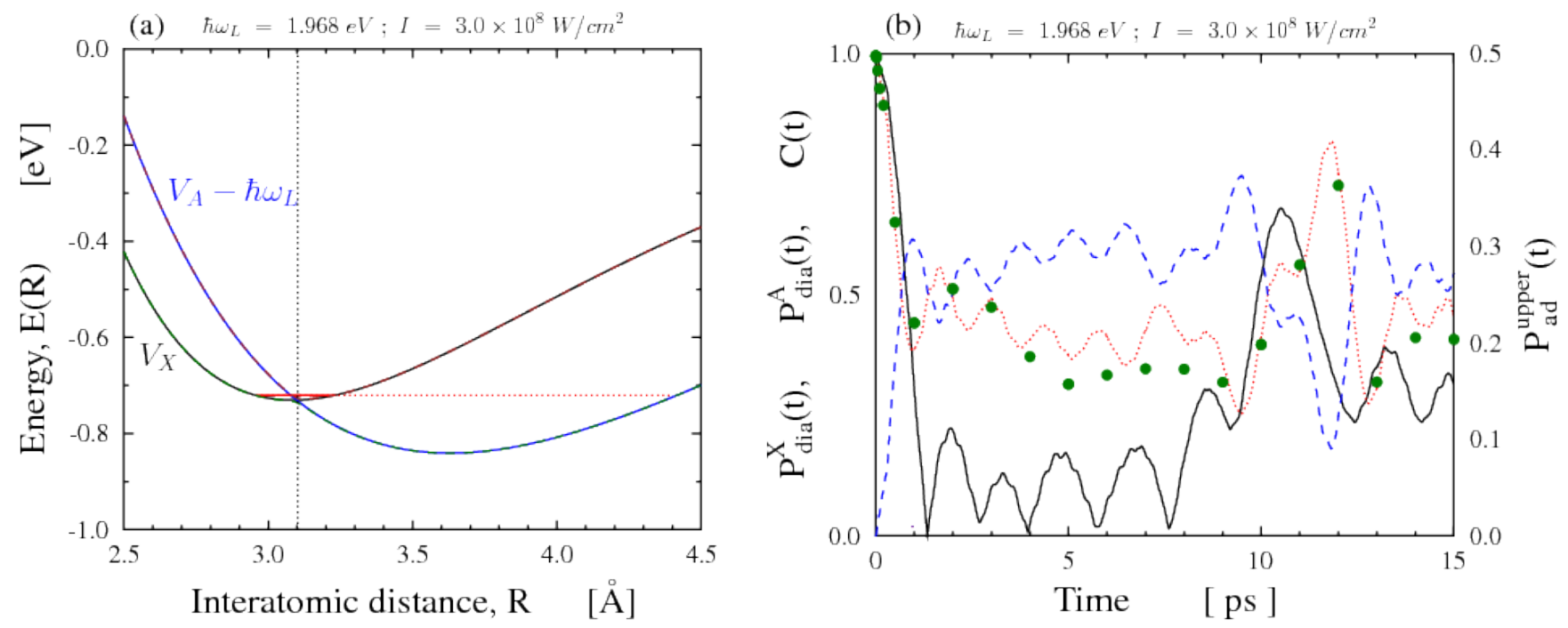

Figure 6: The same as Figure 5 except that the laser frequency is now increased to $\hbar \omega_{L}=1.968 \mathrm{eV}$. For more details see Fig. 5 .

rapidly within the $1^{\text {st }} p s$ and then continues to oscillate around approximately the value of $\sim 0.4$ also exhibiting - as in the case of the autocorrelation function - a local enhancement at around 9.5 ps. A recurrence in the autocorrelation function implies that the wave packet has returned partly to the initial one. Since the initial wave packet in here starts from the ground state X surface, the population on this surface may also grow around the recurrence. The population of the adiabatic upper state follow approximately the shape of that of the X state.

Because of the applied weak field in the cases studied above in Figs. 5 and 6, the energy split between the adiabatic upper and lower curves at the crossing point of the diabatic curves and $\theta=0$ is extremely small and almost negligible (it amounts to $\sim 10 \mathrm{meV}$ ). However, the picture changes markedly in the case of the higher field intensity studied here, $I=3.0 \times 10^{10} \frac{\mathrm{W}}{\mathrm{cm}^{2}}$. These adiabatic curves are depicted in addition to the diabatic curves in panels (a) of Figs. 7 and 8. Increasing the field intensity increases the energy split substantially as expected from the off diagonal term in the potential matrix $\hat{\mathbf{W}}$ in (6). At the value of $R$ of the CI where the respective diabatic curves cross, the energy split is twice the absolute value of this off diagonal term. In polyatomic 
molecules it is known that the appearance of nonadiabatic effects intensifies due to conical intersections when the so-called interstate coupling constants increase [10]. In the present context of light-induced CIs the analogous interstate coupling constant is given by $\epsilon_{0} d(R) / 2$ as can be seen by comparing Eq.(6) and the respective equations in Ref.[10]. Since this interstate coupling constant increases with increasing field intensity, we expect stronger nonadiabatic effects in stronger fields, in general.

Again, as for the weak field, we study here two situation with different laser frequencies. We depict in Fig. 7 the results for the lower frequency $\hbar \omega_{L}=1.851 \mathrm{eV}$. We see that the autocorrelation function now decays very fast within the first $200 \mathrm{fs}$ to a rather low value of about $\sim 0.35$ and continues to drop to about $\sim 0.21$ during the next $400 \mathrm{fs}$. The population on the $\mathrm{X}$ surface is permanently decreasing (from 1 to $\sim 0.55$ ) during the first $600 \mathrm{fs}$. In contrast, the population on the upper adiabatic surface changes very little (see the scale on the right hand side of the figure). This is due to the fact that the initially populated level is well below the energy of the CI and essentially within the lower adiabatic surface, see Fig. 7(a).

The results obtained for the larger laser frequency $\left(\hbar \omega_{L}=1.968 \mathrm{eV}\right)$ are summarized in Fig. 8. The energy of the initial wave packet is now slightly above the energy of the CI and is significantly larger than the bottom of the $V_{A}-\hbar \omega_{L}$ curve. We see that due to this energetic situation the dynamics is pretty fast and the autocorrelation function drops significantly to only about 0.23 within the short time of just $75 \mathrm{fs}$. Later on this function continues to decay exhibiting oscillations. This behavior of the autocorrelation function is rather similar to those found in small polyatomics with field free CIs $[1,3]$. The populations of the diabatic and in this example of the adiabatic states as well also show rapid substantial changes on a similarly short time scale. Interestingly, the population on the diabatic A surface, which is zero initially, now becomes considerably larger than that of the $\mathrm{X}$ surface at most of the time interval depicted in Fig. 8(b).

Finally, we would like to gain some insight into the impact of the CI by freezing the rotational degree of freedom. For this purpose we assume that the orientation of the system is fixed during the process and the molecular axis is parallel to the polarization of the laser field, i.e., we set $\cos \theta=1$ in eq.(6), to maximize the coupling between the electronic states. In what follows we compare the results of this calculations (referred to as the "no CI" case) with the above shown full calculations ("CI" case). The results obtained for the autocorrelation function and the adiabatic population are collected in Fig. 9 for $\hbar \omega_{L}=1.968 \mathrm{eV}$ and $I=3.0 \times 10^{10} \frac{\mathrm{W}}{\mathrm{cm}^{2}}$ where they are also compared with the respective quantities taken from Fig. 8. The differences between the "no CI" and "CI" results are obviously considerable. First, the behavior of the "no Cr" autocorrelation function is rather structured, while the correct function is nearly smooth showing a more or less continuous decay. This differ- ence is due to the much larger density of states available and populated in the "CI" case where many odd J values contribute in contrast to only $\mathrm{J}=0$ in the "no CI" case. Interestingly, the "no CI" autocorrelation function decreases much faster at very short times (up to $30 \mathrm{fs}$ ) than the correct one, and at later times it shows dramatic recurrences up to a value of 0.8 in sharp contrast to the decaying function in the "CI" case.

Another quantity of interest is the population of the upper adiabatic state. Both the "CI" and "no CI" results are rather comparable in the short time period where the autocorrelation function drops fast (about $30 \mathrm{fs}$ ). At longer times, the deviation between the populations become quite remarkable. In the "no CI" case the adiabatic population jumps back close to its original value and then falls off relatively flatly except of the "bump" occurring at around $300 \mathrm{fs}$, a time when the autocorrelation function has its large recurrences. In contrast, the correct population of the upper adiabatic state continues to decay and at the end of the $600 \mathrm{fs}$ shown in Fig. 9 more than $60 \%$ of its initial value has been transferred to the lower adiabatic state.

One may be tempted to expect that if the laser intensity is weak, say $I=3.0 \times 10^{8} \frac{\mathrm{W}}{\mathrm{cm}^{2}}$, the deviations of the "no CI" from the "CI" results will be hardly visible. This is, however, not necessarily the situation. In Fig. 10 we display in analogy to Fig. 9 the results obtained for the weak intensity. The "no CI" results are surprisingly different from the "CI" ones already discussed above. First of all, we notice that like in the stronger field (see Fig. 9 ), also here the autocorrelation function in the "no CI" case decreases more rapidly at the very beginning than in the "CI" case. But the most striking feature of the autocorrelation function in the "no CI" case is the appearance of nearly complete periodic recurrences where the wave packet returns to the initial wave function. We shall return to this behavior below. Concerning the population on the upper adiabatic surface, it seems rather meaningless to compare between the "no CI" and the "CI" cases. The former one displays much more similarities to the autocorrelation function in the "no CI" case than having any resemblance to the result of the "CI" case.

To understand the behavior in the "no CI" case, we should study some details of the actual wave function. It turns out that at the given photon energy accidentally two eigenstates of the field free Hamiltonian $\left(\varphi_{\nu=0}^{X}\right.$ and $\left.\varphi_{\nu=8}^{A}\right)$ are nearly degenerate: $E_{8}^{A}-E_{0}^{X} \approx$ $25 \mu \mathrm{eV}$. In the laser field these two field free states will strongly mix giving rise to the two new eigenstates: $\left|\phi_{522}\right\rangle \approx\left(0.68\left|\varphi_{0}^{X}\right\rangle ; 0.72\left|\varphi_{8}^{A}\right\rangle\right)^{T}$ and $\left|\phi_{552}\right\rangle \approx$ $\left(0.71\left|\varphi_{0}^{X}\right\rangle ;-0.70\left|\varphi_{8}^{A}\right\rangle\right)^{T}$, with which have an energy split of: $E_{552}-E_{522} \approx 3.06 \mathrm{meV}$. Our initial wave function at $t=0$ is the electronic and vibrational ground state of the field free system $\left(\varphi_{\nu=0}^{X}\right)$, and can be excellently expressed by these two eigenstates: $|\Psi(t=0)\rangle=$ $\left(\left|\varphi_{0}^{X}\right\rangle ; 0\right)^{T} \approx 0.68\left|\phi_{522}\right\rangle+0.71\left|\phi_{552}\right\rangle$. At later times we have a beating between these two eigenstates and the 

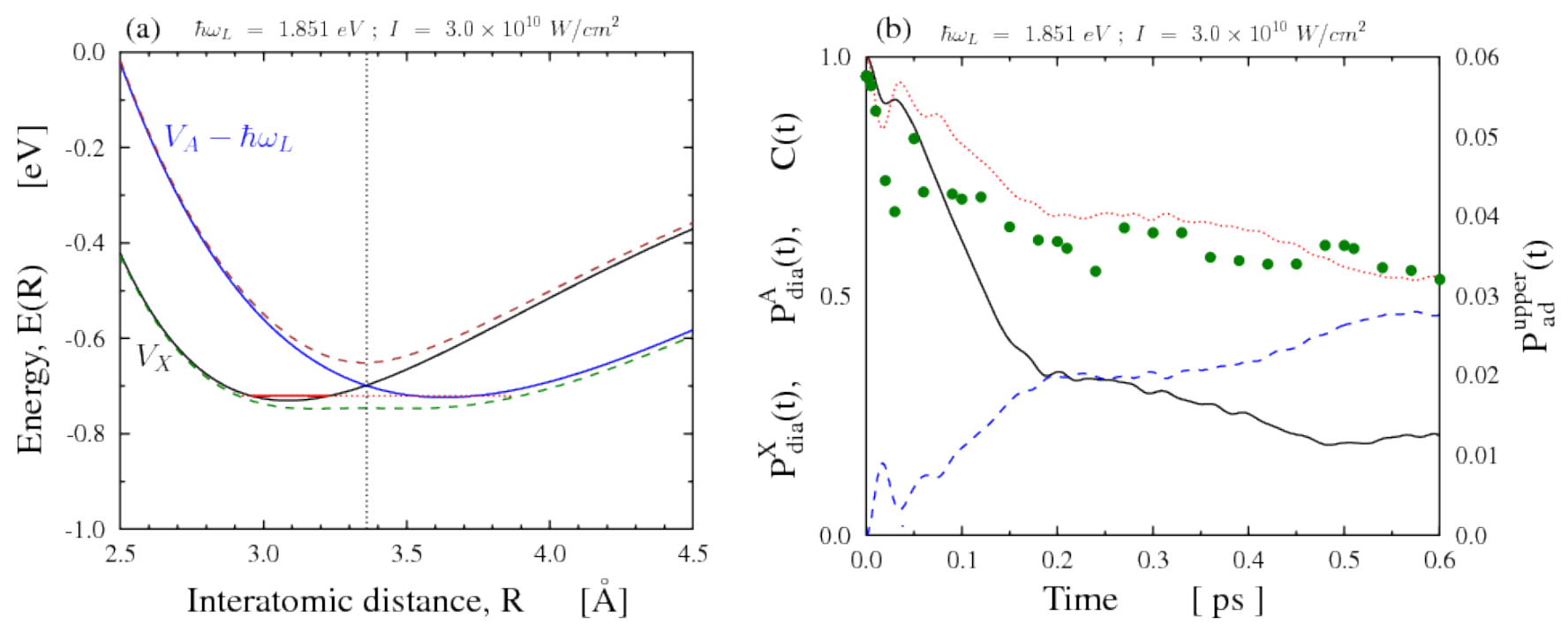

Figure 7: Potential curves and results for field intensity $I=3.0 \times 10^{10} \frac{\mathrm{W}}{\mathrm{cm}^{2}}$ and frequency $\hbar \omega_{L}=1.851 \mathrm{eV}$. (a) Potential energy $V_{X}$ and field-dressed potential energy $V_{A}-\hbar \omega_{L}$ curves of $\mathrm{Na}_{2}$. Shown are also the respective adiabatic potential curves at $\theta=0$ as dashed curves. The dashed-dotted vertical line indicates the geometric position of the CI and the dashed-dotted horizontal line the energy position of the initial state of the propagation. (b) Autocorrelation function (solid line), population on the ground state diabatic surface (dotted line), on the excited state diabatic surface (dashed line) and on the adiabatic upper state surface $(\bullet \bullet \bullet)$ are shown as a function of time.
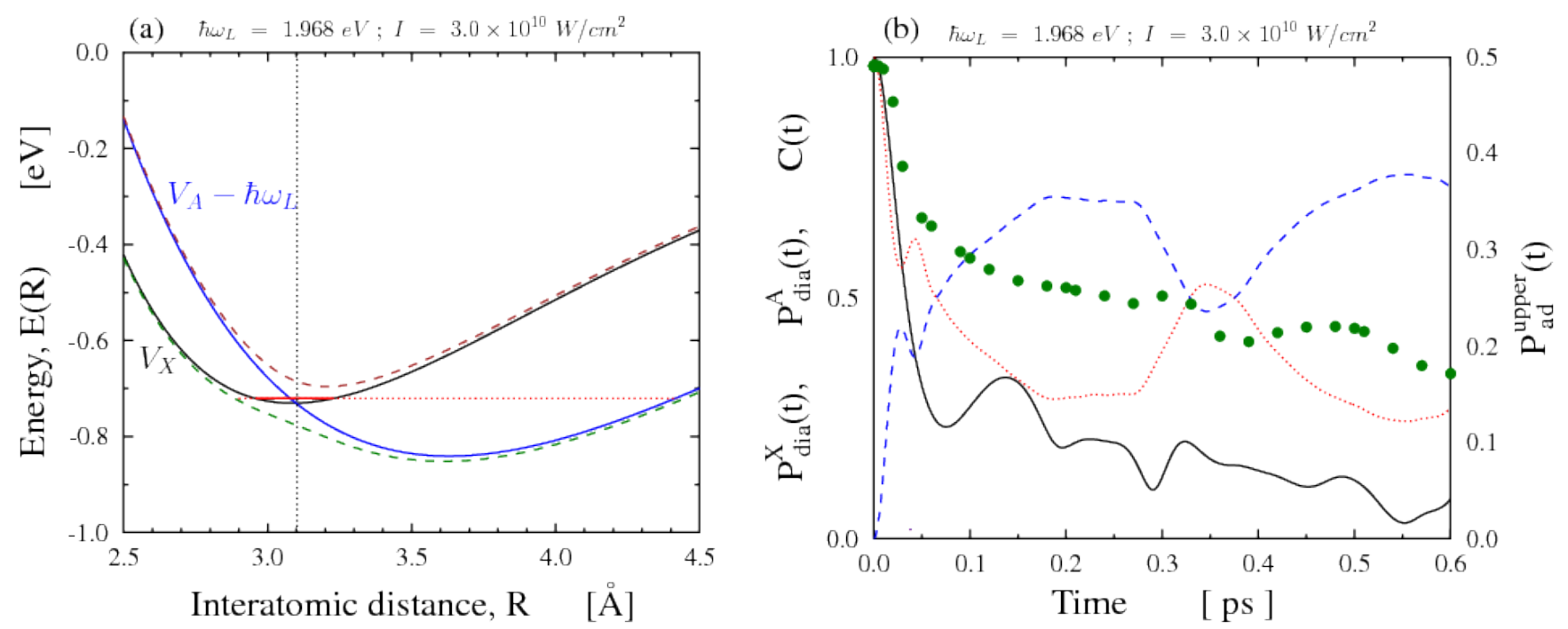

Figure 8: The same as Figure 7 except that the laser frequency is now increased to $\hbar \omega_{L}=1.968 \mathrm{eV}$. For more details see Fig. 7.

energy split of $\sim 3 \mathrm{meV}$ leads to periodic recurrences in the autocorrelation function with a time period of about $T \approx 1.36 \mathrm{ps}$ as observed in the calculations. In the "CI" case the situation is rather different as initial state having $J=0$ couples directly only to field free states of $J=1$ which in turn couple to $J=0$ and 3 states etc. Consequently, the nearly degenerate state which is responsible for the striking beating found in the "no CI" case is irrelevant in the full calculation. To demonstrate the impact of the CI we briefly mention that the starting wave function when expressed by the respective eigen- functions acquires contributions of similar weights from 8 eigenfunctions. Moreover, when expanded in field free states, the eigenfunctions in the " $\mathrm{Cr}$ " case are found to be mixtures of several rotationally excited eigenstates of the field free problem on the two diabatic surfaces with significant coefficients $(>0.2)$ up to $J=13$. 


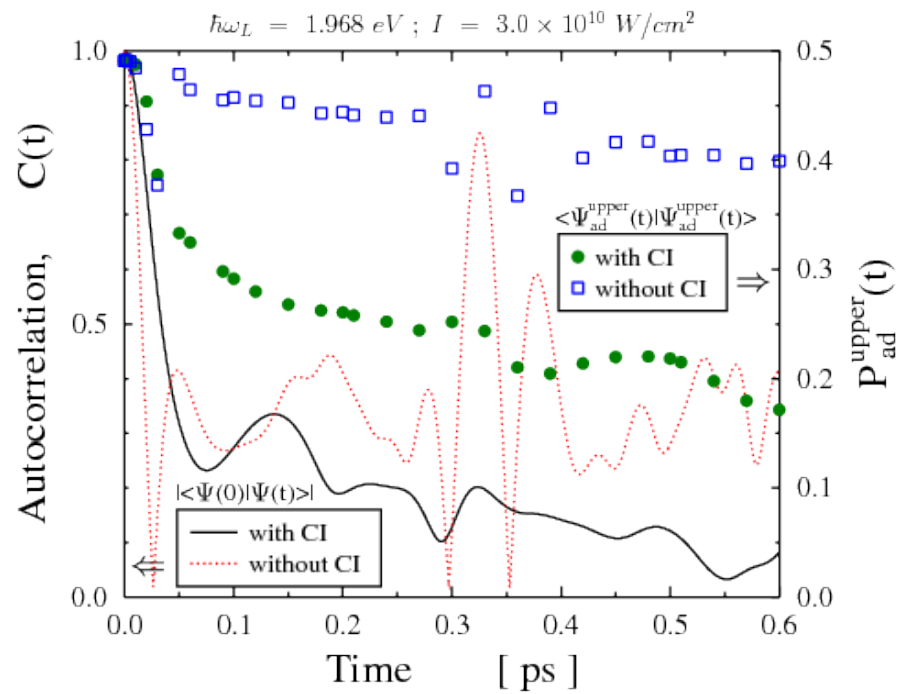

Figure 9: Autocorrelation function and population on the adiabatic upper state surface as a function of time for the "no CI" case where the rotational motion has been frozen $(\cos \theta=1$ in eq.(6)). The results are compared with those of the full calculations ("CI" case). The laser field intensity and photon energy are: $I=3.0 \times 10^{10} \frac{\mathrm{W}}{\mathrm{cm}^{2}}$ and $\hbar \omega_{L}=1.968 \mathrm{eV}$. The curves of the autocorrelation function are marked with (solid line) and (dotted line) for the "CI" and "no CI" cases, respectively. The curves of the upper state population are marked with (••) and ( $\square \square \square \square)$ for the "CI" and "no CI" cases, respectively.

\section{Conclusions}

Recently, a novel and physically interesting phenomenon was found $[13,14]$ in the field of light-matter interactions. It was shown theoretically that exposing a diatomic molecule to a laser field can give rise to the appearance of so called light-induced CIs. In this situation the rotation is the additional nuclear degree of freedom which enables the formation of the CIs. The topology of these light-induced CIs is easily revealed by describing the molecule-light interaction in field-dressed electronic state picture. As is well known from the field of nonadiabatic processes, the nuclear dynamics is strongly coupled to the electronic dynamics in the vicinity of CIs, due to the appearance of infinitely large nonadiabatic coupling terms. Being singular at the CI, the nonadiabatic couplings may become the source of numerous statical and dynamical phenomena. The possibility to induce artificially - by using a laser field - strong nonadiabatic effects into a molecular system promises a wealth of controllable new phenomena even in diatomic molecules.

Conical intersections are abundant in free polyatomic molecules. There is a significant difference between these natural CIs and the laser-induced ones. The natural CIs can not be modified while light-induced ones can be controlled. The positions of these CIs are determined by the laser frequency and the strengths of their nonadiabatic couplings by the intensity of the laser.

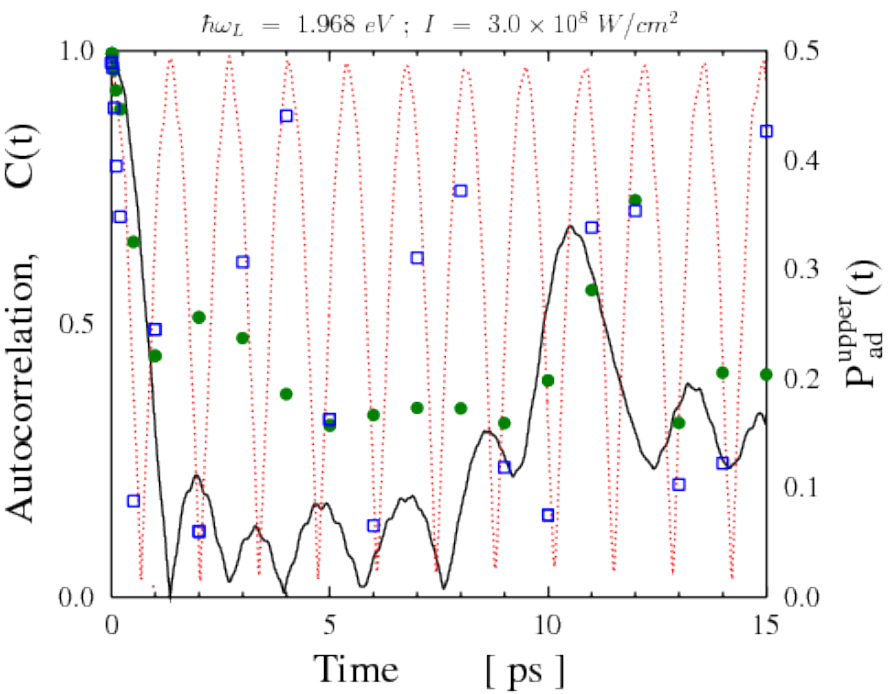

Figure 10: Autocorrelation function and population on the adiabatic upper state surface are figured as a function of time. The applied laser field intensity and photon energy are: $I=$ $3.0 \times 10^{8} \frac{\mathrm{W}}{\mathrm{cm}^{2}}$ and $\hbar \omega_{L}=1.968 \mathrm{eV}$. For more details see Fig. 9 .

In this work the topological or Berry phase in the $\mathrm{Na}_{2}$ molecule was studied. We performed calculations along closed contours that surround a light-induced CI and obtained $\alpha=\pi$ for the value of the topological phase. As known for the case of single CIs given in nature, this result can be considered as a proof that a "true" conical intersection has been found. Interesting results have also been found for contours which do not surround the light-induced CI. At the heart of this work we have also performed wave packet calculations demonstrating that the light-induced CI has a strong impact on the molecular dynamics even for weak laser fields. The situation resembles that in field free polyatomic molecules where the dynamics is highly affected by CIs given by nature owing to the strong coupling between the nuclei and the electrons.

\section{Appendix}

A. On the propagation of a wave packet with a time-dependent Hamiltonian in the Floquet picture

The TDSE we wish to solve is

$$
H(t) \psi(t)=i \hbar \partial_{t} \Psi(t)
$$

where $H(t)=H(t+T)$ stands the time-dependent Hamiltonian for a diatomic molecule in a cw laser field with the frequency $\omega_{L}=2 \pi / T$.

In our studied case

$H(t)=H_{A}(R, \theta)\left|\Psi_{A}^{\text {elec }}\right\rangle\left\langle\Psi_{A}^{\text {elec }}\left|+H_{X}(R, \theta)\right| \Psi_{X}^{\text {elec }}\right\rangle\left\langle\Psi_{X}^{\text {elec }}\right|$ 
$+\mathcal{E}_{0} \cos (\theta) d(R) \cos \left(\omega_{L} t\right) \cdot$

- $\left[\left|\Psi_{A}^{\text {elec }}\right\rangle\left\langle\Psi_{X}^{\text {elec }}|+| \Psi_{X}^{\text {elec }}\right\rangle\left\langle\Psi_{A}^{\text {elec }}\right|\right]$

where $H_{X}$ is the time-independent Hamiltonian with the ground electronic energy as function of $\mathrm{R}$ as a potential and similarly $H_{A}$ is the time-independent Hamiltonian for the first excited electronic state.

The initial state is the field-free state of the molecule which is suddenly exposed to the cw laser beam. In our case the initial state is taken as the ground vibrational and rotational eigenstate of $H_{X}, \chi_{\nu=0, J=0}^{X}$ and therefore,

$$
\left.\left|\Psi(t=0)>=\chi_{\nu=0, J=0}^{X}\right| \Psi_{X}^{\text {elec }}\right\rangle .
$$

Using the Shirley approach [26] we can expand the solution of the TDSE with the quasi-energy (Floquet) solutions which we denote here by $\mid \Psi_{\alpha}^{Q E}(t)>$ (we will explain what they are later). Following Shirley the solution of Eq.18 is given by,

$$
\left|\Psi(t)>=\sum_{\alpha} C_{\alpha} \Psi_{\alpha}^{Q E}(t)\right\rangle
$$

where

$$
C_{\alpha}=\left\langle\Psi_{\alpha}^{Q E}(t=0) \mid \Psi(t=0)\right\rangle
$$

The quasi-energy (Floquet) solutions are defined as (see for example, [27, 28])

$$
\left|\Psi_{\alpha}^{Q E}(t)\right\rangle=e^{-i E_{\alpha}^{Q E} t / \hbar} \sum_{n=-\infty}^{\infty} e^{i \omega_{L} n t}\left|\varphi_{n, \alpha}\right\rangle
$$

where correspondingly $E_{\alpha}^{Q E}$ and the n-th components of the vector $\left|\vec{\varphi}_{\alpha}\right\rangle$ are the eigenvalues and eigen-states of the time-independent Floquet matrix, $\mathcal{H}_{F}$, which its $\left(n, n^{\prime}\right)$ matrix element is defined as

$$
\begin{aligned}
{\left[\mathcal{H}_{F}\right]_{n, n^{\prime}}=} & \frac{1}{T} \int_{0}^{T} e^{-i \omega_{L} n t}\left[-i \hbar \frac{\partial}{\partial t}+H(t)\right] e^{+i \omega_{L} n^{\prime} t} d t
\end{aligned}
$$

where $\left\{\left(n^{\prime}, n\right)\right\}_{0, \pm 1, \pm 2, \ldots}$. By substituting Eq.23 into Eq.21 (see the (t,t') formalism as derived by [29]) one gets that the solution of the TDSE is given by

$$
\mid \Psi(t)>=\sum_{n} e^{-i \omega_{L} n t}\left[e^{-i \mathcal{H}_{F} t / \hbar} \mid \Psi(t=0)>\right]_{n, n^{\prime}=0}
$$

and therefore when $\left\{e^{-i \omega_{L} n t}\right\}_{n=0, \pm 1, \ldots}$ are used as a basis set then

$$
\mathcal{H}_{F} \vec{\psi}(t)=i \hbar \frac{d}{d t} \vec{\psi}(t)
$$

where each one of the Fourier component is the fieldfree initial state. For our case the $\left(n, n^{\prime}\right)$ Floquet matrix element is defined as

$$
\begin{aligned}
{\left[\mathcal{H}_{F}\right]_{n^{\prime}, n} } & =\delta_{2 n^{\prime}, 2 n} H_{X}(R, \theta)\left|\Psi_{X}^{\text {elec }}\right\rangle\left\langle\Psi_{X}^{\text {elec }}\right| \\
& +\delta_{2 n^{\prime}+1,2 n+1} H_{A}(R, \theta)\left|\Psi_{A}^{\text {elec }}\right\rangle\left\langle\Psi_{A}^{\text {elec }}\right| \\
& +\left[\delta_{2 n^{\prime}, 2 n+1}+\delta_{2 n+1,2 n^{\prime}}\right] \frac{\mathcal{E}_{0}}{2} \cos (\theta) d(R) . \\
& \cdot\left[\left|\Psi_{A}^{\text {elec }}\right\rangle\left\langle\Psi_{X}^{\text {elec }}|+| \Psi_{X}^{\text {elec }}\right\rangle\left\langle\Psi_{A}^{\text {elec }}\right|\right]
\end{aligned}
$$

Even when the laser field is strong the Fourier components can be truncated and the Floquet matrix can be truncated. For the case where the photoinduced dynamics is a net one-photon process (as in our case) it is enough to take $\left\{n^{\prime}, n\right\}=0,-1$ or $\left\{n^{\prime}, n\right\}=0,+1$ and the initial state is the field-free state which is defined above in Eq.22. For our studied case the time-independent Floquet matrix which is given above in Eq.27 and the propagation of the wavepacket within the framework of the Floquet formalism as given above on Eq.26 are as defined in Eqs.1-6 in our paper.

\section{Acknowledgment}

Á.V. acknowledges the OTKA Grant No. 80095. The work is partly supported by TÁMOP 4.2 .1 ./B$09 / 1 / \mathrm{KONV}-2010-0007 / \mathrm{IK} / \mathrm{IT}$ project. The project is implemented through the New Hungary Development Plan co-financed by the European Social Fund, and the European Regional Development Fund. N.M. acknowledged ISF 96/07. L.S.C. acknowledges financial support by the DFG.
[1] W. Domcke, D. R. Yarkony, H. Köppel, Eds.; Conical Intersections: Electronic Structure, Dynamics and Spectroscopy; World Scientific: Singapore, (2004).

[2] M. Baer, Beyond Born Oppenheimer: Electronic NonAdiabatic Coupling Terms and Conical Intersections; Wiley: Hoboken, NJ, (2006).

[3] G. A. Worth, L. S. Cederbaum, Annu. Rev. Phys. Chem. 55, 127 (2004).

[4] M. Klessinger, J. Michl, Excited States and Photochemistry of Organic Molecules; VCH Publishers Inc,; New York, (1995).
[5] P. Kukura, et al. Science 310, 1006 (2005).

[6] S. Hahn, G. J. Stock, Phys. Chem. B. 104, 1146 (2000).

[7] T. Schultz, et al. Science 306, 1765 (2004).

[8] Z. Lan, L. M. Frutos, A. Sobolewski, W. Domcke, Proc. Natl. Acad. Sci. 105, 12707 (2008).

[9] T. Andruniow, N. Ferre, M. Olivucci, Proc. Natl. Acad. Sci. 101, 17908 (2004).

[10] H. Köppel, W. Domcke, L. S. Cederbaum, Adv. Chem. Phys. 57, 59 (1984).

[11] M. Baer, G. D. Billing, The Role of Degenerate States in Chemistry, Advances in Chemical Physics Vol. 124 
Wiley-Interscience, New York, (2002).

[12] S. Matsika, Rev. Comp. Chem. 23, 83 (2007).

[13] N. Moiseyev, M. Sindelka, L. S. Cederbaum, J. Phys B: At. Mol. Opt. Phys. 41, 221001 (2008).

[14] M. Sindelka, N. Moiseyev, L. S. Cederbaum, J. Phys B: At. Mol. Opt. Phys. 44, 045603 (2011).

[15] R. Lefebvre, O. Atabek, M. Sindelka, and N. Moiseyev, Phys. Rev. Lett., 103, 123003, (2009).

[16] V. S. Prabhudesai, U. Lev, A. Natan et al., Phys. Rev. A, 81, 023401, (2010).

[17] O. Atabek and G. Jolicard, Phys. Rev. A, 49, 1186, (1994); O. Atabek, R. Lefebvre, and F. X. Gadéa, Phys. Rev. A, 74, 063412, (2006).

[18] R. Numico, A. Keller, and O. Atabek, Phys. Rev. A 52, 1298 (1995).

[19] E. Charron, A. Giusti-Suzor, and F. H. Mies, Phys. Rev. Lett., 75, 2815, (1995).

[20] M. Baer, Physics Reports 358, 75 (2002).

[21] G. Herzberg, H. C. Longuet-Higgins, Discuss. Faraday Soc. 35, 77 (1963).

[22] H. C. Longuet-Higgins, Proc. R. Soc. London, Ser. A 344, 147 (1975).

[23] M. V. Berry, Proc. R. Soc. London A 392, 45 (1984).

[24] S. Magnier et al., J. Chem. Phys. 98, 7113 (1993); W. T. Zemke et al., J. Mol. Spectrosc., 85, 150 (1981).

[25] C. A. Mead, D. G. Truhlar, J. Chem. Phys., 70, 2284 (1979).

[26] J. H. Shirley, Phys. Rev. B 138, 979 (1965).

[27] F. H. M. Faisal, Theory of Multiphoton Processes, Plenum, New York, (1986).

[28] N. Moiseyev, Non-hermitian Quantum Mechanics, Cambridge University Press, Cambridge, (2011).

[29] U. Peskin, N. Moiseyev, J. Chem. Phys., 99, 4590 (1993).

[30] Throughout the article the autocorrelation function and the diabatic populations are calculated directly from the time dependent coefficients of the expansion of the wave packet in our basis functions (field free eigenfunctions). The population on the adiabatic (upper) surface is numerically integrated over the configuration space $(R, \theta)$ at only of the time points marked in the figures and its values are given by the scale on the right side of the figures.

Black \& white version of Figure 4:
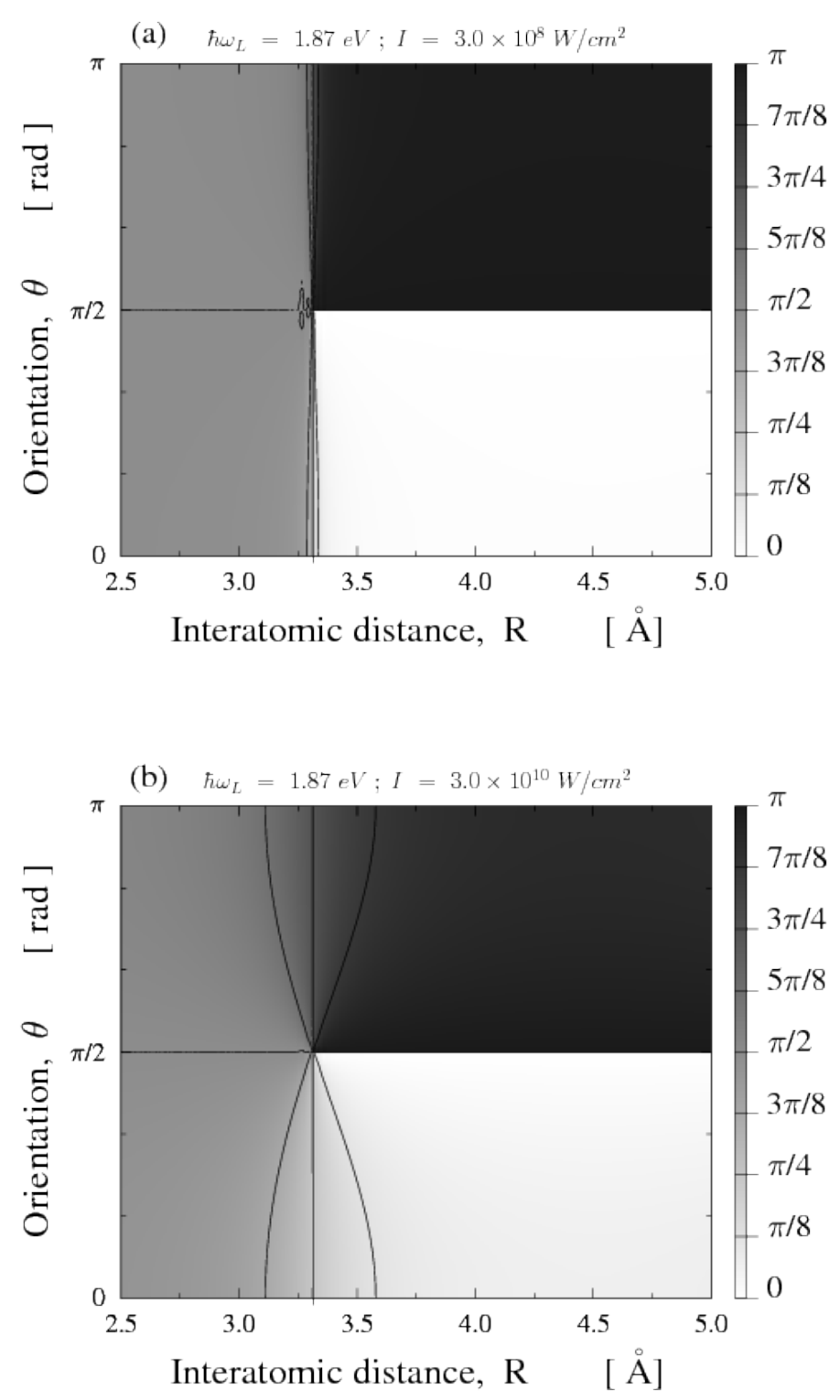